\title{
Configuration design of collective housing building structure - IFD systems configuration
}

\author{
J.Nikolic \\ UPC (Universidad Politècnica de Catalunya), Department of Architectural Construction I, Barcelona, \\ Spain
}

\begin{abstract}
Residential buildings are still based on conventional construction process where components and subsystems are parts of closed-static systems, based on fixed connections and mixed functions. Uncontrolled overlapping of different functions in the building structure creates a strong dependency conditions between building layout and technical systems. Labyrinth of interfaces and fixed joints create complex relations between elements, and don't support transformations. The design of building structure as integrated, flexible and demountable systems configuration will determine a systems disassembly and deconstruction rather than demolition. Systems configuration based on independent and exchangeable components and subsystems applied for more permanent building parts (load-bearing structure, partitions and façade) may become the solution for the new dynamics in residential building life, applying flexible and demountable (IFD) systems configuration for the different levels of technical integration, with the special emphases on the potential benefit of "Kit of parts" integrated systems.
\end{abstract}

\section{INTRODUCTION}

\subsection{Present Mass Housing solid model}

Main problem of contemporary housing today is in the strongly dependences of functions and elements by fixed joints into closed configuration. Principal part of the static buildings corresponds to its structure. The structure is defined as a configuration model of four principal functions and corresponding technical systems: load-bearing, enclosing, interior partitioning and building installations network system, where elements are put together into disordered and mixed relations by fixed connections. For that reason the transformation at the building level is related with time consuming by long transformation processes, significant energy and material lose and waste production. Main characteristic of massive structure are:

- Functional dependency with two or more functions integrated into one component;

- Closed assemblies of components into rigid connections.

- Zero "flexibility" of floor plan distribution: also the interior panels formed part of the crossbearing supporting concrete structure;

- Elements are not interchangeable, but fixed together without possibility to be repair and replaced.

- Services' equipment, electric cables and installations pipes and ducts are embedded into the structural and partitions walls.

In the Figure 1 is shown the conventional building as mixed system of different levels. Loadbearing partition walls deal with more functions: load-bearing and partitioning and are fixed together in load-bearing structure. The same occurs with façade panel. Different functional levels are closely dependent because one component is used for more functions. Different technical levels are also dependent by fixed connections between components. 


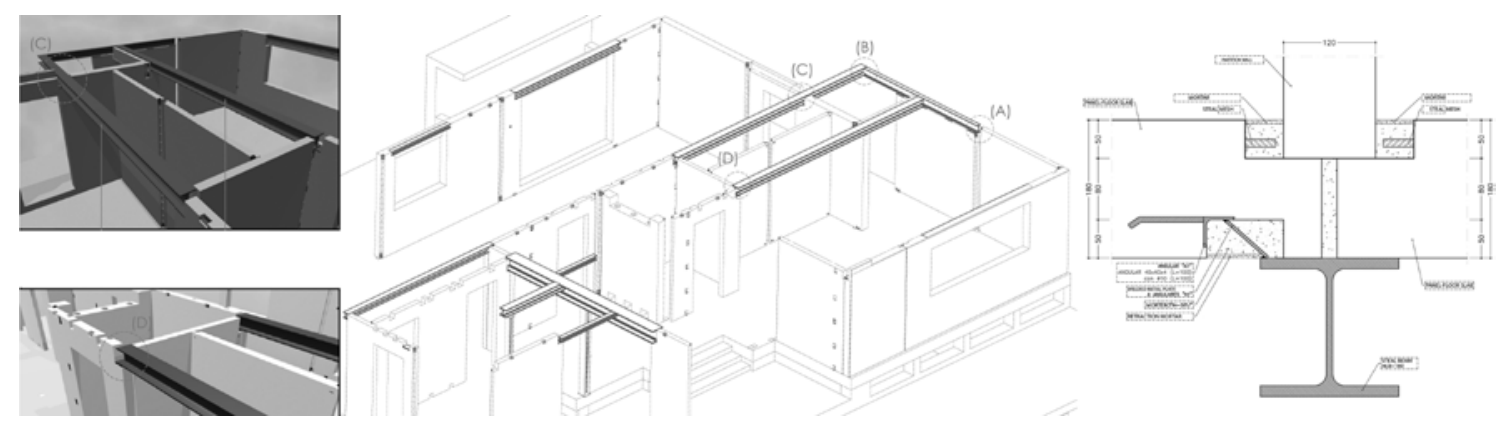

Figure 1. Functional dependency in building structure by permanent connections of different construction material (precast concrete and steel) in social housing building in Granollers, Spain, 2007

The strong dependency between building parts and rigid connections are the boundary condition for transformations and adaptability of integrated structure. The building is considered inflexible and its configuration is considered "closed"- static. In building industrialization, industrial product may be considered in two ways: i) the industrial product is entire building; the industrial products are building parts, systems, components. The first case is developed as an industrial method of solid models. When the building layout doesn't work according to occupants' requirements and load-bearing partitions can't be rearranged, inevitable changes in building layout affect the integrity of slower changing functional levels (load bearing structure) because the partitions deal with more functions (partitioning and load-bearing). Changes in the building layout are directly related to the technical composition of the building structure. Adaptability in the building layout is difficult to achieve if:

- Interfaces between the components in the configuration, are not designed as demountable.

- The two or more functions are integrated in one building component.

- There is a large number of relations between components within the system.

- The elements are closely related to each other creating closed hierarchies.

- High complicity of in-between connection.

In order to avoid this dependences, technical levels such building structure, should be independent. Assembling and disassembling of building structure composed from elements, should be defined through the different types of independent levels. Levels may be defined according to the changes of studied aspects (functional, technical, spatial, material...). The theory of levels introduces systematization of elements into number of independent assemblies and group assemblies, and relations between the fast changing and slow changing components and systems.

\subsection{From solid model to systems configuration}

For the transformable high-rice apartment building the existing tools, applied in the building process need to be updated for more disassembly options and building transformations according to different requirements.

Every new phase in building use implies new requirements for spatial organization. This involves transformations of the building and its technical configuration and means that after each use phase an "assessment"-configuration-assembly should be designed and built to indicate the structure suitability for the new spatial requirements. Spatial, technical and physical characteristics of structural configuration based on elements and the relations between them is analyzed to describe Integrated, Flexible and Demountable - IFD systems configuration for building structure. Technical aspects refers to what elements are put together in connections and how; spatial refers to how the structure is designed and its spatial characteristics; and physical deals with materials and the type of connection between different materials. Flexible systems configuration is a model that can respond to different requirements for changes of spatial systems and technical systems. Spatial transformations describe changes in the building layout and technical refer to systems' transformations and upgrading. All subsystems and components are assembled to allow maximum flexibility in the dwelling layout. From the other side, the sub-systems will be considered adaptable, exchangeable, and compatible to generate flexibility for configuration trans- 
formations and upgrading. Demountable systems configuration is a system capable for major reconfiguration, to be dismantled and rebuilt somewhere else. Demountable system is "open" and "flexible" where components and subsystems are joined by dry and simple connections to support reconfiguration of the building without demolitions.

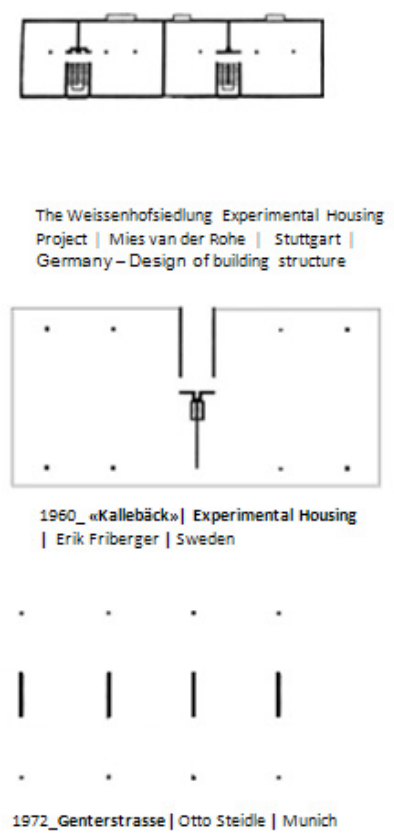

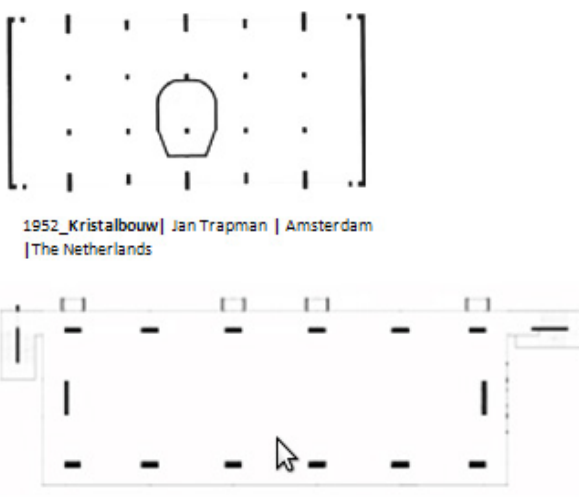

2001_uFlexsus House 22w| Takenaka Corporation

Japa

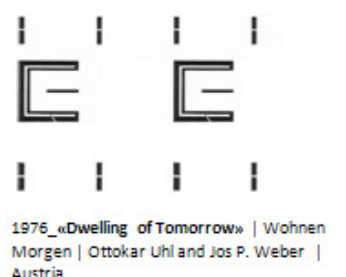

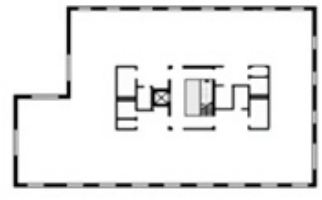

2003 _osiedlung Hegianwandweg * EM2N Architekten | Switzerland

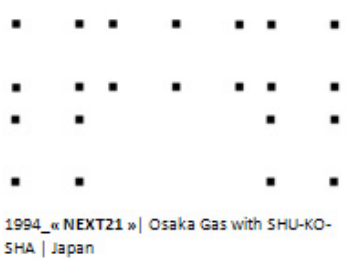
1994_ENEXT
SHA | Japan

Figure 2. The best practices of Open Residential project: Flexible layout of building structure

\subsection{Open Building Initiatives}

One of the most urgent issues of Residential Open Building from the 60' was the "in-built" flexibility of the floor plan for spatial transformations. The main issue of Open Building approach was a design and construction of building "support" with special focus on load-bearing structure as independent technical and functional level. Load-bearing structure of social apartment buildings was designed and built with maximum flexibility to support transformations in the building layout. Principal characteristics of flexible load-bearing structure were: i) long span structures, ii) middle span area free from the load-bearing elements, iii) one-directional loadbearing construction, not cross-bearing; v) demountable dry joints. The best flexible configuration models for "support" structure showed in the Figure 2 present the layouts of flexible loadbearing structure for multiunit building. Building structure is designed and constructed as flexible and/or demountable configuration composed from industrial systems and components. Independency is mainly important between technical systems that correspond to different functions. New network aspects should support the systematization of industrial systems and components into integrated, flexible and demountable IFD systems configuration. Industrialized construction techniques should support precise high quality details in connections; flexible configuration model allows adaptability of spatial and technical systems and changes at various levels of integration and demountable properties meet the needs for configuration transformations by disassembly and re-configuration. Building structure that supports spatial and technical transformations according to different requirements will be considered open systems configuration.

\subsection{Technical levels of control}

Theory of levels is the base for the IFD systems configuration that recognize different parts of the building with different life cycles. In the building design are introduced levels of technical composition, which corresponds to the main building functions. The life circle of one set of 
function-construction relationships becomes shorter because of rapid functional change and static configuration set. To integrated structural configuration composed from different components, subsystems and systems, building structure should be defined through the different independent levels.

\section{METODOLOGY}

The main issue of sustainable construction is to find the balance between dynamics in use, adaptability and changes in the housing layout, economy, and the fundamental principles of environmental ecology. Transformation capacity of building structure is considered as a principal condition for building adaptability. Systematization of industrialized components and subsystems by coordinated relations to achieve flexible and demountable configuration of industrialized elements is based on elements assembly into independent technical levels. The arrangement of the components and systems into independent technical and functional levels and demountable-dry joints support IFD systems configuration approach. Analysis of the recent apartment buildings in Europe has been done according to open building principles and the main findings will be used to highlight a new tendency toward IFD collective housing. This research analyze "El Polvorí" social housing building built in Barcelona by Pich Aguilera Architects in 2005, to be considered as IFD project with special focus on: i) configuration design of building structure; ii) integrated solutions by demountable and dry joints; iii) independence and exchangeability aspects in the structural configuration; iv) adaptability progression in the network between spatial and technical systems to be achieved. This research will show that the transformation capacity of total building strongly depends on the transformation capacity of building configuration as technical system of different subsystems and components and the way they are put in connections. The study focuses on the assembling and connecting of building parts with the special focus on the demountable characteristics of the independent and dependent elements to get better understanding about the way they interface, in order to facilitate refurbishment while minimizing waste.

\section{BUILDING STRUCTURE CONFIGURATION MODEL}

\subsection{Structural adaptability: new integrated approach which is flexible and demountable}

The main parts of the building system are its sub-systems. Subsystem is a set of predetermined, standardized, industrial components. If all subsystem's components are put together by fixed connections the configuration is "closed"-“static". Building system composed from independent subsystem is "open" system. The open system can exchange parts, components and subsystems outside its original production contest. Interchangeable components and subsystems offer an opportunity for many manufactures to participate in the systems configuration. Industrialized systems configuration can offer increased adaptability to changes through the precise jointing features of the factory-made components and sub-systems. Since most factory-made components or sub-systems are designed to facilitate site installation, they could also be dismantled to generate change without any demolition.

Systematization of the sub-systems and components in one systems configuration correspond to the main structural functions: load-bearing, enclosing, partitioning and servicing. According to main functions are established corresponding technical levels: load-bearing structure, envelope, partitions and services. Systematization of elements into main technical levels is based on hierarchy assemblies where components and materials make connections by simple and demountable dry joints. The hierarchy assemblies define the tree systematic path where elements lower in the hierarchy will change with more frequency. Demountable joints allow for the fast changing components to be independent and replaced at the end of life. Open system that can exchange parts, components and subsystems outside its original production contest are considered "interchangeable" and compatible. Interchangeable components and subsystems offer an opportunity for many manufactures to participate in the systems configuration design 
and future re-configuration. Exchangeability of components between different manufactures are possible if their interface geometry are compatible.

\subsection{Demountable system configuration applied for load-bearing structure: Case study of "El Polvorí" social housing}

"El Polvorí" collective housing is a set of eight new housing blocks of dwellings for the integration of families from the old buildings that existed before in the same location. Industrial components, systems and techniques were used for building structure. The design and construction are based on systematization of the load-bearing structure and cladding of the blocks. Among the architects and engineers has been done the design of the configuration for the bearing structure. The key element of the system is the "three-dimensional module" that is structurally rigid core of the building and architectural space for bathrooms and kitchens. 3D modules contain each home services allowing maximum flexibility for the distribution of the interiors. The load-bearing structure is completed by the columns and beams that form the front porches, and hollow core floor slabs.

Key design tasks were to synthesize a number of subsystems and their components into one integrated configuration model based on demountable connections. An integrated configuration for building structure is assembled from a series of independent components. This decomposition into the subsystems and components allows for a load-bearing structure that is technicallyflexible, where fast changing components can be easily replaced. Assembly and disassembly of components into more complex configuration levels is achieved by simple and demountable bolted joints. Main IFD systems configuration development strategies of "El Polvorí" structure are:

- Systematization of structural components into independent technical level, according to the main functions;

- Integration of various subsystems and components to optimize design and operation process according to different technical levels;

- Use of the „Kit of parts“ structural systems configuration.

- Use of simple dry joints for reduction of dependencies between structural elements and simplification of the construction.

- Design of building layout without load-bearing partitions;

- Achievement of indeterminate space with larger span and open plan allowing non-load bearing partitions to be installed in and removed according to user needs;

- Reduction of non-adaptable or non-accessible services;

- Physical separation of building components by their duration years.

\subsection{Integrated, Flexible and Demountable IFD systems configuration}

Building Structure is integrated systems configuration composed of three independent subsystems: i) 3D modules - customized, prefabricated components for particular design, ii) IFD frames from precast concrete columns and beams, iii) hollow core floor slabs. This integrated "Kit of parts" system is composed of beams, columns and slabs as standardized components and “customized” 3D modules (Fig.3)
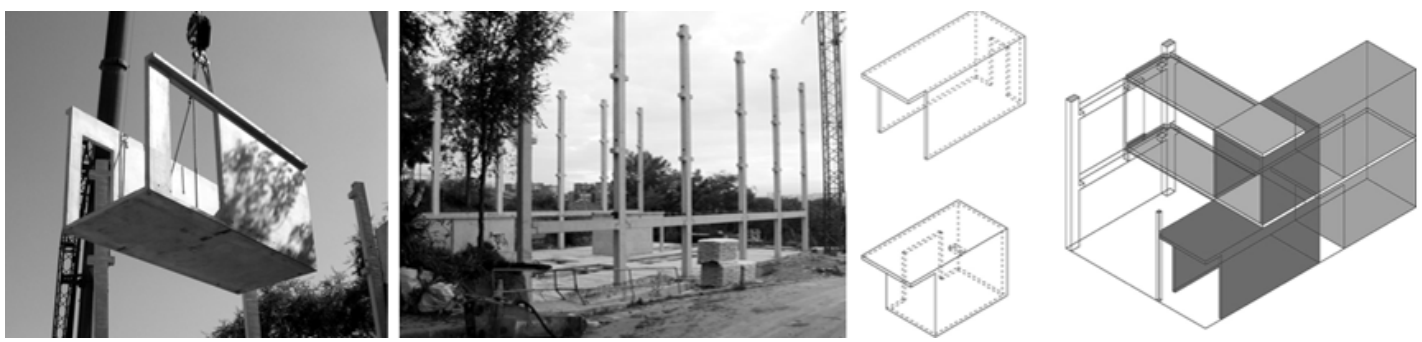

Figure 3. "El Polvorí" structural components: 3D module, column-beam frame, IFD configuration model 
The decomposition by components or, by components assemblies and simple dry joints between them allow for a configuration that is technologically flexible. Industrialized components such as cladding panels, floor slabs, columns and beams can be easily replaced by disassembly process. Exchangeability and independence of components allow transformations at the loadbearing level. 3D modules are customized components with all mechanical, electrical and plumbing duct and wirings for bathrooms and kitchens, preinstalled into the modules' walls. The hollow-core slabs are supported from one side by 3D modules and from the other by concrete column-beam frames. Between this two supports is generated the „open plan“ layout for each dwelling.

Clear separation of functions at the design level is used to support independency and exchangeability of subsystems and components. Dwellings' HVAC pipes and ducts are permanently installed into 3D modules and make the only exception from demountable configuration model. Technologically flexible system supports both: the state-of-the-art of the construction industry and its innovation by exchangeable components at the level of building structure and "infill" partitions and user's requirements for adaptability of leaving space.

In the case "El Polvorí" building structure is design and built as en integrated, flexible and demountable systems configuration composed from three independent subsystems. "Integrated" is the new term to describe compound configuration model for building structure, from components and subsystems produced from different manufactures and put together by simple and demountable dry joints. Demountable systems configuration contributes to reducing initial construction and life cycle costs in various ways:

- By using industrialized construction components, the site job is minimized and waste during construction can be avoided;

- During a building's occupancy and use, independent subsystems allow easy replacement of components:

- After a building's life, the disassembly of its component stands for the reuse and recycling of parts.

\subsection{How the IFD systems configuration works?}

Main work managed by IFD systems configuration are structural transformations at different levels of technical integration. The IFD systems configuration transformation- $T$ can be defined as a sum of independent components or subsystems transformations (equation 1)

$$
T=t 1+t 2+t 3+t 4+t 5+\ldots+t n .
$$

$$
\text { n- number of integrated elements }
$$

Building transformation $\mathrm{T}$ is constant when the building structure is permanent system but designed to be flexible for the dwelling unit transformations. On the Figure 2 are shown the best flexible layouts of load-bearing structure as the independent technical level. Load-bearing structure is considered flexible configuration, when is possible to transform systems configuration.

$$
\mathrm{T}(\text { variable })=\mathrm{t} 1+\mathrm{t} 2+\mathrm{t} 3+\mathrm{t} 4+\mathrm{t} 5+\ldots+\mathrm{tn}
$$

n- number of individual components and systems transformations

"Kit-of-parts" term refers to the study and application of systems configuration, where components are pre-designed / pre-engineered /pre-fabricated to be installed in joint-based configurations. Kit-of-parts demountable system is a special subset of components and evidently is the most appropriate type of construction for the systems configuration sets that attempts to achieve flexibility in assembly, possible collaboration with the construction in situ or other components' producers and efficiency in achieving transformation of the building structure. In the IFD Kit- 
of-parts configuration model different elements can be assembled and taken apart in a variety of ways.

\section{CONCLUSIONS}

This research introduces the building structure as integrated, flexible and demountable configuration composed of industrialized components and subsystems systematized into independent technical levels according to main building functions: load-bearing, partitioning, enclosing and servicing. Technical levels represent integration of functional levels and allow easy access for fast cycling components that change more rapidly. Building structure design according to independent technical levels help to identify elements that have different life span and to coordinate structure assembly process for flexibility in the building layout. Main principles for building structure configuration design:

- "Configuration design" position, dimensions, location, composition, connections of systems and components is based on independent spatial, technical and material levels.

- Load-bearing structure as demountable systems configuration model based on simple and demountable dry joints.

- Flexible distribution of the construction elements in the building layout allows for dwelling unit a number of different distributions in the building layout.

- Possibility to change the surface of the floor plan, either by additional construction or changes in the boundaries of units out of the structure limits.

- The adaptability of structural configuration could be achieved by disassembly and reconfiguration.

- Connection between load-bearing components in load-bearing system is based on simple dryjoints.

- Connections between removable parts and load-bearing structure are based on simple dry joints.

- Position of services and access to them to be independent subsystems.

Finally, dynamic building structure designed and constructed for flexibility and disassembly on spatial, technical and material levels could be considered as a main issue for housing industry to offer innovative approaches for more sustainable and green buildings. IFD systems configuration supports the special and technical building transformations according to systematization of components into independent technical and functional levels. Building transformations represents a dynamic behavior of the components and subsystems in different technical levels to be repair or replaced at the end of their life cycle. One way to approach this problem is through the new design strategy of the housing building as demountable systems building that will support the dynamic behavior of buildings on spatial, structural and material level. New configuration design characteristics for dynamic building systems will be applied for building in general and especially for the "support" structure as the main boundary condition for transformations.

\section{REFERENCES}

Brand, S. 1994. How Buildings Learn: What Happens after They're Built. New York: Viking Press.

Durmisevic, E. 2006. Transformable building structures: Design for disassembly as a way to introduce sustainable engineering to a building design and construction. Delft: Printed by cedris M\&CC, ISBN-10, TU Delft.

Habraken, N.J. 1976. Variation: The Systematic Design of Supports. Laboratory of Architecture and Planing, MIT. USA: Cambridge Mass.

Kieran, S. \& Timberlake, J. 2004. Refabricating Architecture: How manufacturing methodologies are poised to transform building construction. NY: McGraw Hill.

Kendal, S. \& Teicher , J. 2000. Residential Open Building. London \& NY: III.,E \& FN Spon, ISBN 0419-23830.

Durmisevic, E. 2001. From massive construction to decomposition of housing - a way to support further industrialization and customization of housing. Proceedings from the IAHS Conference on Housing. 
Gibb, A.A. \& Sparksman, W.G. \& Neale, R.H. 1999. Standardization and pre-assembly adding value to construction projects. Construction Industry Research and Information Association (CIRIA), Report R176, pp. 220, ISBN 0860174980.

Nikolic, J. 2012. Development of Industrialized, Flexible and Demountable Multifamily Buildings: IFD Colective Housing. Proceedings from the 7th International Conference on Innovation in Architecture, Engineering and Construction, AEC201. Sao Paulo 15-17 August 2012.

Salas, J. 2008. De los sistemas de prefabricación cerrada a la industrialización sutil de la edificación: algunas claves del cambio tecnológico. Informes de la construcción, Vol. 60, 512, 19-34. Instituto de Ciencias de la Construcción Eduardo Torroja (CSIC). Octubre-Noviembre 2008. Madrid. 